\title{
Association of Cardiac Troponin T Concentration on Admission with Prognosis in Critically III Patients without Myocardial Infarction: A Cohort Study
}

\section{Ruijie Xie* \\ Qingui Chen (1)* \\ Wanmei He \\ Mian Zeng}

Department of Medical Intensive Care Unit, The First Affiliated Hospital, Sun Yat-sen University, Guangzhou, Guangdong, People's Republic of China

*These authors contributed equally to this work
Correspondence: Mian Zeng

Department of Medical Intensive Care Unit, The First Affiliated Hospital, Sun

Yat-sen University, No. 58 Zhongshan

Road 2, Guangzhou, Guangdong, 510080,

People's Republic of China

Tel +86-20-87755766

Email zengmian@mail.sysu.edu.cn
Purpose: To investigate the association of cardiac Troponin T (cTnT) with prognosis in critically ill patients without myocardial infarction.

Methods: Adult patients admitted to the intensive care units (ICUs) of the Beth Israel Deaconess Medical Center between 2008 and 2019 who were free of myocardial infarction with a length of ICU stay $\geq 24$ hours and available cTnT records within 24 hours before and after ICU admission were included. The association between cTnT on ICU admission and hospital mortality was evaluated by multivariable logistic regression analysis. The discrimination capacity of cTnT on ICU admission for predicting hospital mortality was examined by receiver operating characteristic (ROC) analysis.

Results: A total of 2960 patients were included. Elevated cTnT $(>0.01 \mathrm{ng} / \mathrm{mL})$ was observed in $2730(92.23 \%)$ patients with a higher hospital mortality compared to normal cTnT (11.21\% versus $7.39 \%, \mathrm{P}=0.075$ ). There was no statistically significant association between elevated cTnT on ICU admission and hospital mortality (adjusted odds ratio 1.50, 95\% confidence interval (CI) $0.88-2.57$ ). Poor discrimination capacity was found for cTnT on ICU admission to predict hospital mortality (area under the ROC curve $0.48,95 \%$ CI 0.44 $0.53)$.

Conclusion: cTnT on ICU admission has limited prognostic value in critically ill patients without myocardial infarction.

Keywords: cardiac troponin T, prognosis, critical care

\section{Introduction}

Cardiac Troponin (cTn) $\mathrm{T}$ is one of the subunits of cTn complex which regulates the contraction of striated muscle. ${ }^{1}$ Due to its tissue-specific expression (ie, cardiac myocyte), cTnT has become a useful biochemical marker for the diagnosis of myocardial infarction. ${ }^{2}$ The main cellular mechanism is that cTn including cTnT would be released into the circulation and become detectable when myocytes are irreversibly damaged. ${ }^{3}$ However, the elevation of cTn is not always caused by acute coronary syndromes. A variety of other conditions may also lead to the elevation, including sepsis, ${ }^{4}$ atrial fibrillation, ${ }^{5}$ heart failure, ${ }^{6}$ exacerbation of chronic obstructive pulmonary disease, ${ }^{7}$ and chronic kidney disease, ${ }^{8}$ behind which the potential cellular mechanism is related to increased myocyte membrane permeability. ${ }^{1,4}$ The prognostic information provided by cTn further supports its usefulness in patients 
with acute coronary syndromes, which can help frame therapeutic decisions, ${ }^{1,9}$ and this may also be applied to patients without acute coronary syndromes. A metaanalysis included about 154,052 participants suggests high cTnT concentration (within the normal range) is associated with increased cardiovascular disease risk in the healthy general population. ${ }^{10} \mathrm{~A}$ similar association is observed in patients with both type 2 diabetes and stable ischemic heart disease. ${ }^{11}$ However, this association has not been well examined in critically ill patients without acute coronary syndromes. Since the above-mentioned many causes of cTn elevation are prevalent in critically ill patients, the elevation of $\mathrm{cTn}$ is common in this patient population, but currently evidence about the association between cTn elevation and prognosis is very limited (especially for cTnT). A meta-analysis included 4,492 critically ill patients from 23 studies reports that cTn elevation is found in $21 \%-59 \%$ of the patients, and elevated cTn is significantly associated with an increased risk of death and an increased length of intensive care unit (ICU) stay. ${ }^{12}$ It should be noted that most of these studies were with a very limited sample size (ie, less than 300), and the three studies which had larger sample sizes (ie, about 1,000) only studied cTnI. ${ }^{13-15}$ In this study, we included a larger cohort of critically ill patients without myocardial infarction and investigated the association between cTnT concentration on ICU admission and prognosis.

\section{Methods}

\section{Data Source and Ethical Information}

The study used data obtained from Medical Information Mart for Intensive Care IV (MIMIC IV, version 0.4), ${ }^{16}$ a database containing comprehensive information (including vital signs, laboratory measurements, diagnosis, administered medications) for each patient admitted to ICUs of a tertiary academic medical center in the United States, the Beth Israel Deaconess Medical Center, between 2008 and 2019.

The database is released under the Health Insurance Portability and Accountability Act (HIPAA) safe harbor provision. Access to the database was approved after completing the Collaborative Institutional Training Initiative (CITI) "Data or Specimens Only Research" course. This study was exempt from institutional review board approval and patient consent due to the retrospective design, lack of direct patient intervention, and the security schema for the re-identification risk. The study complied with the Declaration of Helsinki.

Codes from the code repository mimic-iv (https:// github.com/MIT-LCP/mimic-iv) were used for data extraction. For identification of diseases, International Classification of Diseases, Ninth Revision, Clinical Modification (ICD-9-CM) codes or International Classification of Diseases, Tenth Revision, Clinical Modification (ICD-10-CM) codes were used.

\section{Study Population}

The study used a cohort study design and included adult ICU patients in the database with available measurement records of cTnT. In detail, all patients admitted to one of the ICUs of the Beth Israel Deaconess Medical Center between 2008 and 2019 were screened, but only the first ICU admission of the first hospitalization in the database were further examined if a patient had multiple admission records in the database. Only adult ( $\geq 18$ years) patients with a length of ICU stay $\geq 24$ hours (according to the first ICU hospitalization if a patient had more than one ICU admission during the hospitalization) would be included. Patients who had no measurement records of cTnT within 24 hours before and after the date of ICU admission (according to the first ICU hospitalization if a patient had more than one ICU admission during the hospitalization), or who had a diagnosis record of myocardial infarction (either prevalent or old myocardial infarction, identified by ICD-9-CM codes 410, 412 and ICD-10-CM codes I21, I22, I252) would be excluded. To exclude potential data error, patients whose lengths of (complete) hospitalization were shorter than the lengths of ICU stay (according to the first ICU hospitalization if a patient had more than one ICU admission during the hospitalization) would also be excluded. Figure 1 presents the selection of the study population.

\section{Exposure}

The exposure of the study was cTnT concentration (in serum or plasma, which was not clearly stated in the database) on ICU admission assessed by a standard troponin $\mathrm{T}$ assay (Logical Observation Identifiers Names and Codes (LOINC) code 6598-7; lower reference limit $0 \mathrm{ng} / \mathrm{mL}$, and upper reference limit $0.01 \mathrm{ng} / \mathrm{mL}$ ). For patients who had more than one measurement record of cTnT within 24 hours before and after the date of ICU admission (according to the first ICU hospitalization if a patient had more than one ICU admission during the hospitalization), the maximum 


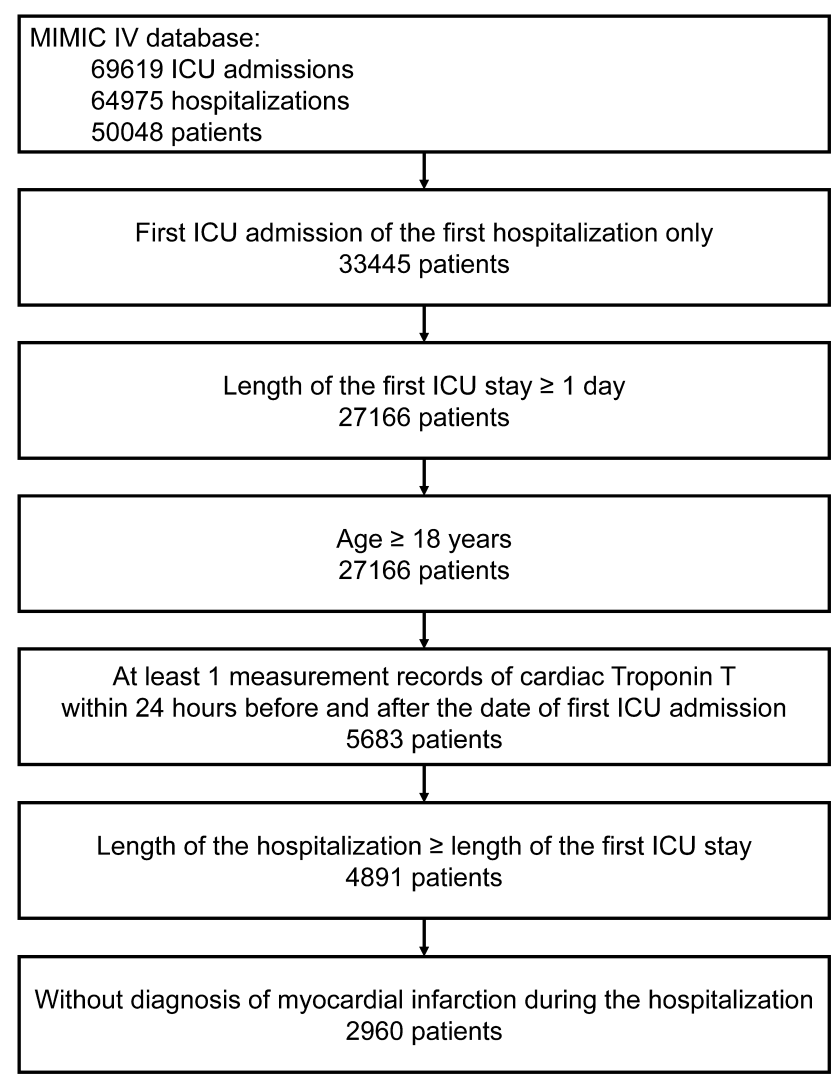

Figure I Selection of the study population. Abbreviation: ICU, intensive care unit.

concentration would be studied as the exposure. Instead of being studied as a continuous variable, cTnT concentration was treated as a dichotomous variable (ie, normal $(\leq 0.01 \mathrm{ng} /$ $\mathrm{mL}$ ) or abnormal $(>0.01 \mathrm{ng} / \mathrm{mL})$ ) or a categorical variable with five levels (using $0.01 \mathrm{ng} / \mathrm{mL}$, and the 25th, 50th, and 75th percentiles of cTnT concentration in the patients with abnormal cTnT concentration $(>0.01 \mathrm{ng} / \mathrm{mL})$ as cut-offs).

\section{Outcomes}

The included patients were followed from the date of ICU admission to the date of hospital discharge for the main study outcome hospital mortality. Length of hospital stay and length of ICU stay (according to the first ICU hospitalization if a patient had more than one ICU admission during the hospitalization) were also studied as outcomes, but the analyses for these two outcomes were merely descriptive.

\section{Covariates}

The date of ICU admission (according to the first ICU hospitalization if a patient had more than one ICU admission during the hospitalization) was considered as the baseline for each included patient. The following variables at baseline were studied as covariates in the study: age, sex, ethnicity, types of ICU (according to the first ICU hospitalization if a patient had more than one ICU admission during the hospitalization), Simplified Acute Physiology Score II (SAPS II), Glasgow Coma Scale (GCS), Sequential Organ Failure Assessment (SOFA), Charlson Comorbidity Index (CCI), ${ }^{17}$ and dialysis. In addition to $\mathrm{CCI}$, the below comorbidities were also identified based on diagnoses made during the complete hospitalization: congestive heart failure, peripheral vascular disease, cerebrovascular disease, dementia, chronic pulmonary disease, rheumatic disease, peptic ulcer disease, mild liver disease, diabetes without complication, diabetes with complication, paraplegia, renal disease, malignant cancer, severe liver disease, metastatic solid tumor, and acquired immunodeficiency syndrome.

\section{Statistical Analysis}

Continuous variables are presented as median (25th-75th percentile) and categorical variables are presented as number (percentage). Comparisons between groups were examined by Kruskal-Wallis $H$-test for continuous variables and Chi-squared test or Fisher's exact test for categorical variables. The relationship between cTnT concentration and hospital mortality was plotted based on a univariable logistic regression model. Multivariable logistic regression models were used to evaluate the association between the exposure and hospital mortality. Two models were planned: Model 1 was adjusting for age, sex, ethnicity, SAPS II, and CCI; Model 2 was adjusting for Model 1 plus types of ICU. Receiver operating characteristic (ROC) curve was plotted and area under the ROC curve (AUC) was calculated to evaluate the discrimination capacity of cTnT concentration for predicting hospital mortality. The bootstrap method (resampling times $=500$ ) was used to estimate the $95 \%$ confidence intervals (CIs) of AUC. A P value less than 0.05 was considered to indicate statistical significance. Empower(R) (www.empowerstats.com; X\&Y solutions, Inc., Boston, MA, USA) and R software, version 3.4.3 (http://www.r-project.org; R Foundation for Statistical Computing, Vienna, Austria) were used for statistical analyses.

\section{Results}

\section{Patient Characteristics}

A total of 2,960 patients were included with a median age of 71 (25th-75th percentile 59-82) years and 56.93\% were 
male. Most of the patients were admitted to Medical Intensive Care Unit (26.05\%), Coronary Care Unit (21.66\%), Medical/Surgical Intensive Care Unit (16.15\%), and Surgical Intensive Care Unit (13.99\%). The most prevalent comorbidities were congestive heart failure (30.54\%), chronic pulmonary disease (21.93\%), diabetes without complication $(20.20 \%)$, renal disease (20.07\%), and cerebrovascular disease (15.98\%).

Elevated cTnT concentration $(>0.01 \mathrm{ng} / \mathrm{mL})$ was observed in $92.23 \%(2,730 / 2,960)$ of the patients (median $0.08,25$ th- 75 th percentile $0.04-0.26 \mathrm{ng} / \mathrm{mL}$ ). There was no statistically significant difference in age, sex, and ethnicity between patients with and without elevated cTnT concentration, but patients with elevated cTnT concentration had a higher proportion of being admitted Coronary Care Unit $(22.71 \%$ versus $9.13 \%, \mathrm{P}<0.001)$ together with a higher prevalence of congestive heart failure $(31.21 \%$ versus $22.61 \%, \mathrm{P}=0.007)$. Patients with elevated cTnT concentration also showed higher SAPS II (38 versus 36, $\mathrm{P}=0.026)$, lower GCS (14 (25th-75th percentile 10-15) versus 14 (25th-75th percentile 11-15), $\mathrm{P}=0.041$ ), and higher SOFA ( 5 versus $4, \mathrm{P}<0.001$ ) on ICU admission when compared with patients with normal cTnT concentration. Detailed baseline characteristics of the study population are presented in Table 1.

\section{Prognosis}

The overall hospital mortality for the study population was $10.91 \%(323 / 2,960)$, with a median length of hospital stay of 8.64 (25th-75th percentile 5.23-14.84) days and a median length of ICU stay of 3.22 (25th-75th percentile 1.91-6.78) days. Compared with patients with normal cTnT concentration, patients with elevated cTnT concentration $(>0.01 \mathrm{ng} / \mathrm{mL})$ had a higher hospital mortality (11.21\% versus $7.39 \%, \mathrm{P}=0.075)$, a longer length of hospital stay (8.69 days versus 7.91 days, $\mathrm{P}=0.160$ ), and a longer length of ICU stay (3.27 days versus 2.92 days, $\mathrm{P}=0.002)$. When stratified as five categories according to cTnT concentration $(\leq 0.01,0.02-0.04,0.05-0.08,0.09-$ 0.26 , and $\geq 0.27 \mathrm{ng} / \mathrm{mL}$ ), it could be observed that hospital mortality rates and lengths of either hospitalization or ICU stay increased with the increases of $\mathrm{cTnT}$ concentration when the cTnT concentration was less than $0.08 \mathrm{ng} / \mathrm{mL}$, after which the pattern changed to be in a reverse direction, but similar to the results of the analysis which stratified the study population into two categories (ie, $\leq 0.01$ $\mathrm{ng} / \mathrm{mL}$ and $>0.01 \mathrm{ng} / \mathrm{mL}$ ), only the difference in lengths of
ICU stay between categories was statistically significant (Table 2).

\section{Association Between cTnT and Hospital Mortality}

When plotted based on a univariable logistic regression model (Figure 2), it could be observed that the probability of hospital mortality increased with the increase of baseline cTnT concentration. The crude odds ratio (OR) for hospital mortality in patients with elevated cTnT concentration $(>0.01 \mathrm{ng} / \mathrm{mL})$ on ICU admission was $1.58(95 \%$ CI 0.95-2.63) compared to those with normal cTnT concentration. After adjusting for age, sex, ethnicity, SAPS II, CCI, and types of ICU, there was no statistically significant association between elevated cTnT concentration on ICU admission and hospital mortality (OR 1.50, 95\% CI $0.88-2.57)$. When the study population was stratified as five categories according to cTnT concentration $(\leq 0.01$, $0.02-0.04,0.05-0.08,0.09-0.26$, and $\geq 0.27 \mathrm{ng} / \mathrm{mL})$, the largest OR was observed in the stratum $0.05-0.08 \mathrm{ng} / \mathrm{mL}$ (adjusted OR 1.71, 95\% CI 0.95-3.09) when compared to the normal stratum $(\leq 0.01 \mathrm{ng} / \mathrm{mL})$, but no statistically significant associations were observed between the strata (Table 3).

\section{Discrimination Capacity for cTnT to Predict Hospital Mortality}

As presented in Figure 3, poor discrimination capacity was found for cTnT concentration on ICU admission to predict hospital mortality (AUC 0.48, 95\% CI 0.44-0.53). The best threshold was $0.045 \mathrm{ng} / \mathrm{mL}$ based on the maximum value of the Youden's index, with a specificity of $36.59 \%$, a sensitivity $66.56 \%$, and an accuracy $39.86 \%$.

\section{Discussion}

In the study by employing a large cohort (nearly 3,000) of critically ill patients in the absence of myocardial infarction and taking potential confounding into account, we investigated the association between cTnT concentration on ICU admission and prognosis. The main findings of our study include: 1) elevated cTnT concentration is common in ICU patients on admission (ie, $92.23 \%$ in our study); 2) patients with elevated cTnT concentration appeared to have a higher hospital mortality rate and longer lengths of hospitalization and ICU stay when compared with patients with normal cTnT concentration, but only the difference in length of ICU stay is statistically 
Table I Baseline Characteristics of the Study Population

\begin{tabular}{|c|c|c|c|c|}
\hline \multirow[t]{2}{*}{ Variable } & \multirow[t]{2}{*}{$\begin{array}{c}\text { Overall } \\
(\mathrm{N}=2960)\end{array}$} & \multicolumn{2}{|c|}{$\begin{array}{l}\text { Cardiac Troponin T Concentration on } \\
\text { ICU Admission* }\end{array}$} & \multirow[t]{2}{*}{$\mathbf{P}$} \\
\hline & & $\begin{array}{c}\text { Normal } \\
(\leq 0.01 \mathrm{ng} / \mathrm{mL}) \\
(\mathrm{N}=\mathbf{2 3 0})\end{array}$ & $\begin{array}{c}\text { Abnormal } \\
(>0.01 \mathrm{ng} / \mathrm{mL}) \\
(\mathrm{N}=2730)\end{array}$ & \\
\hline Age (years) & 7I (59-82) & $74(59.25-82)$ & 71 (59-82) & 0.352 \\
\hline Sex & & & & 0.169 \\
\hline Male & 1685 (56.93\%) & $12 \mid(52.61 \%)$ & I 564 (57.29\%) & \\
\hline Female & I 275 (43.07\%) & $109(47.39 \%)$ & II 66 (42.7I\%) & \\
\hline Ethnicity & & & & 0.844 \\
\hline White & I94I (65.57\%) & $153(66.52 \%)$ & 1788 (65.49\%) & \\
\hline Black/African American & $232(7.84 \%)$ & $19(8.26 \%)$ & $213(7.80 \%)$ & \\
\hline Hispanic/Latino & $66(2.23 \%)$ & $5(2.17 \%)$ & 61 (2.23\%) & \\
\hline Asian & $72(2.43 \%)$ & $3(1.30 \%)$ & $69(2.53 \%)$ & \\
\hline Other/Unknown & 649 (21.93\%) & $50(21.74 \%)$ & 599 (21.94\%) & \\
\hline Types of ICU** & & & & $<0.001$ \\
\hline Coronary Care Unit & 641 (21.66\%) & $21(9.13 \%)$ & 620 (22.71\%) & \\
\hline Medical Intensive Care Unit & 771 (26.05\%) & $67(29.13 \%)$ & $704(25.79 \%)$ & \\
\hline Medical/Surgical Intensive Care Unit & $478(16.15 \%)$ & $42(18.26 \%)$ & 436 (I5.97\%) & \\
\hline Cardiac Vascular Intensive Care Unit & 247 (8.34\%) & 14 (6.09\%) & $233(8.53 \%)$ & \\
\hline Surgical Intensive Care Unit & $4 \mid 4(13.99 \%)$ & $42(18.26 \%)$ & $372(13.63 \%)$ & \\
\hline Trauma Surgical Intensive Care Unit & $310(10.47 \%)$ & $33(14.35 \%)$ & 277 (10.15\%) & \\
\hline Neuro Surgical Intensive Care Unit & 59 (1.99\%) & $6(2.61 \%)$ & $53(1.94 \%)$ & \\
\hline Neuro Intermediate & 27 (0.9l\%) & $3(1.30 \%)$ & $24(0.88 \%)$ & \\
\hline Neuro Stepdown & $12(0.4 \mid \%)$ & $2(0.87 \%)$ & $10(0.37 \%)$ & \\
\hline Post-anesthesia Care Unit & I (0.03\%) & $0(0.00 \%)$ & I (0.04\%) & \\
\hline SAPS II on ICU admission & $38(30-48)$ & $36(29.25-44)$ & $38(30-48)$ & 0.026 \\
\hline GCS on ICU admission & $14(10-15)$ & $14(11-15)$ & $14(10-15)$ & 0.041 \\
\hline SOFA on ICU admission & $5(3-9)$ & $4(2-6)$ & $5(3-9)$ & $<0.001$ \\
\hline Charlson Comorbidity Index*** & $5(4-7)$ & $5(4-7)$ & $5(3-7)$ & 0.552 \\
\hline Dialysis on ICU admission & 175 (5.9l\%) & $3(1.30 \%)$ & $172(6.30 \%)$ & 0.002 \\
\hline Cardiac Troponin T concentration on ICU admission ( $\mathrm{ng} / \mathrm{mL})^{*}$ & $0.07(0.03-0.23)$ & $0.01(0.01-0.01)$ & $0.08(0.04-0.26)$ & - \\
\hline$\leq 0.01$ & $230(7.77 \%)$ & $230(100.00 \%)$ & - & \\
\hline $0.02-0.04$ & $843(28.48 \%)$ & - & $843(30.88 \%)$ & \\
\hline $0.05-0.08$ & $526(17.77 \%)$ & - & $526(19.27 \%)$ & \\
\hline $0.09-0.26$ & $686(23.18 \%)$ & - & $686(25.13 \%)$ & \\
\hline$\geq 0.27$ & $675(22.80 \%)$ & - & $675(24.73 \%)$ & \\
\hline \multicolumn{5}{|l|}{ Comorbidity*** } \\
\hline Congestive heart failure & 904 (30.54\%) & $52(22.61 \%)$ & $852(31.21 \%)$ & 0.007 \\
\hline Peripheral vascular disease & $245(8.28 \%)$ & $22(9.57 \%)$ & $223(8.17 \%)$ & 0.460 \\
\hline Cerebrovascular disease & $473(15.98 \%)$ & $46(20.00 \%)$ & 427 (15.64\%) & 0.083 \\
\hline Dementia & II 4 (3.85\%) & $12(5.22 \%)$ & $102(3.74 \%)$ & 0.262 \\
\hline Chronic pulmonary disease & 649 (21.93\%) & 47 (20.43\%) & $602(22.05 \%)$ & 0.569 \\
\hline Rheumatic disease & 88 (2.97\%) & 7 (3.04\%) & 81 (2.97\%) & 0.948 \\
\hline Peptic ulcer disease & 61 (2.06\%) & $9(3.91 \%)$ & $52(1.90 \%)$ & 0.040 \\
\hline Mild liver disease & 277 (9.36\%) & 20 (8.70\%) & 257 (9.41\%) & 0.719 \\
\hline
\end{tabular}


Table I (Continued).

\begin{tabular}{|c|c|c|c|c|}
\hline \multirow[t]{2}{*}{ Variable } & \multirow[t]{2}{*}{$\begin{array}{c}\text { Overall } \\
(\mathrm{N}=2960)\end{array}$} & \multicolumn{2}{|c|}{$\begin{array}{l}\text { Cardiac Troponin T Concentration on } \\
\text { ICU Admission* }\end{array}$} & \multirow[t]{2}{*}{$\mathbf{P}$} \\
\hline & & $\begin{array}{c}\text { Normal } \\
(\leq 0.01 \mathrm{ng} / \mathrm{mL}) \\
(\mathrm{N}=\mathbf{2 3 0})\end{array}$ & $\begin{array}{c}\text { Abnormal } \\
(>0.01 \mathrm{ng} / \mathrm{mL}) \\
(\mathrm{N}=\mathbf{2 7 3 0})\end{array}$ & \\
\hline Diabetes without complication & $598(20.20 \%)$ & 55 (23.91\%) & $543(19.89 \%)$ & 0.144 \\
\hline Diabetes with complication & $225(7.60 \%)$ & 17 (7.39\%) & $208(7.62 \%)$ & 0.900 \\
\hline Paraplegia & $173(5.84 \%)$ & 14 (6.09\%) & $159(5.82 \%)$ & 0.870 \\
\hline Renal disease & 594 (20.07\%) & $33(14.35 \%)$ & $56 \mathrm{I}(20.55 \%)$ & 0.024 \\
\hline Malignant cancer & $256(8.65 \%)$ & $25(10.87 \%)$ & $231(8.46 \%)$ & 0.212 \\
\hline Severe liver disease & 86 (2.91\%) & 7 (3.04\%) & $79(2.89 \%)$ & 0.897 \\
\hline Metastatic solid tumor & $108(3.65 \%)$ & $5(2.17 \%)$ & $103(3.77 \%)$ & 0.214 \\
\hline Acquired immunodeficiency syndrome & $4(0.14 \%)$ & I (0.43\%) & $3(0.11 \%)$ & 0.277 \\
\hline
\end{tabular}

Notes: *The maximum cardiac Troponin T concentration of available measurement records of cardiac Troponin T concentration examined within 24 hours before and after the date of first ICU admission; **The first ICU admission if a patient had more than I ICU admission during the hospitalization; ***Calculated or screened based on all diagnosis records made during the hospitalization.

Abbreviations: ICU, intensive care unit; SAPS II, Simplified Acute Physiology Score II; GCS, Glasgow Coma Scale; SOFA, Sequential Organ Failure Assessment.

significant; 3) after adjusting for potential confounding there is no statistically significant association between cTnT concentration on ICU admission and hospital mortality; 4) cTnT concentration on ICU admission has poor discrimination capacity for predicting hospital mortality. Our study provides new evidence and insight into the association between cTnT concentration and prognosis in critically ill patients without myocardial infarction, a patient population in which the association has not been well examined. The findings suggest that cTnT concentration on ICU admission measured by a standard troponin $\mathrm{T}$ assay has limited prognostic value in critically ill patients without myocardial infarction, and therefore in practice this biomarker may be not useful for prognostic risk stratification in this patient population.

Currently, there are few studies that specifically investigated the association between cTnT concentration on ICU admission and prognosis in critically ill patients without myocardial infarction, although a few available studies $^{12}$ with small sample sizes investigated this association in the general ICU patients (ie, including patients with myocardial infarction). Spies et $\mathrm{al}^{18}$ investigated 26 sepsis patients in a surgical ICU and found about $70 \%$ $(18 / 26)$ of the patients had elevated cTnT concentration

Table 2 Prognosis of the Study Population According to Concentration of Cardiac Troponin T on ICU Admission

\begin{tabular}{|c|c|c|c|c|}
\hline $\begin{array}{l}\text { Concentration of Cardiac Troponin } \\
\text { T on ICU Admission* }\end{array}$ & $\begin{array}{r}\text { Cardiac Troponin } \\
\text { T Concentration }(\mathrm{ng} / \mathrm{mL})^{*}\end{array}$ & $\begin{array}{l}\text { Hospital } \\
\text { Mortality }\end{array}$ & $\begin{array}{r}\text { Length of Hospital } \\
\text { Stay (Days) }\end{array}$ & $\begin{array}{r}\text { Length of ICU } \\
\text { Stay (Days)** }\end{array}$ \\
\hline \multicolumn{5}{|l|}{ Two categories (ng/mL) } \\
\hline$\leq 0.01$ (normal) & $0.0 \mathrm{I}(0.0 \mathrm{I}-0.0 \mathrm{I})$ & 17 (7.39\%) & 7.91 (4.92-12.91) & $2.92(1.74-5.13)$ \\
\hline$>0.01$ (abnormal) & $0.08(0.04-0.26)$ & 306 (11.21\%) & $8.69(5.25-14.98)$ & $3.27(1.93-6.93)$ \\
\hline $\mathrm{P}$ & - & 0.075 & 0.160 & 0.002 \\
\hline \multicolumn{5}{|l|}{ Five categories $(\mathrm{ng} / \mathrm{mL})$} \\
\hline$\leq 0.01$ & $0.0 \mathrm{I}(0.0 \mathrm{I}-0.0 \mathrm{I})$ & 17 (7.39\%) & 7.91 (4.92-12.91) & $2.92(1.74-5.13)$ \\
\hline $0.02-0.04$ & $0.03(0.02-0.04)$ & 91 (10.79\%) & $8.05(4.99-14.80)$ & $2.99(1.86-6.16)$ \\
\hline $0.05-0.08$ & $0.06(0.05-0.07)$ & $69(13.12 \%)$ & $9.54(5.75-15.08)$ & $3.65(2.00-7.25)$ \\
\hline $0.09-0.26$ & $0.14(0.11-0.19)$ & 78 (II.37\%) & $8.56(5.32-14.69)$ & $3.10(1.88-6.68)$ \\
\hline$\geq 0.27$ & $0.62(0.38-1.33)$ & $68(10.07 \%)$ & 8.91 (5.24-15.42) & $3.67(2.08-8.00)$ \\
\hline $\mathrm{P}$ & - & 0.184 & 0.136 & $<0.001$ \\
\hline
\end{tabular}

Notes: *The maximum cardiac Troponin T concentration of available measurement records of cardiac Troponin T concentration examined within 24 hours before and after the date of first ICU admission; **The first ICU admission if a patient had more than I ICU admission during the hospitalization.

Abbreviation: ICU, intensive care unit. 

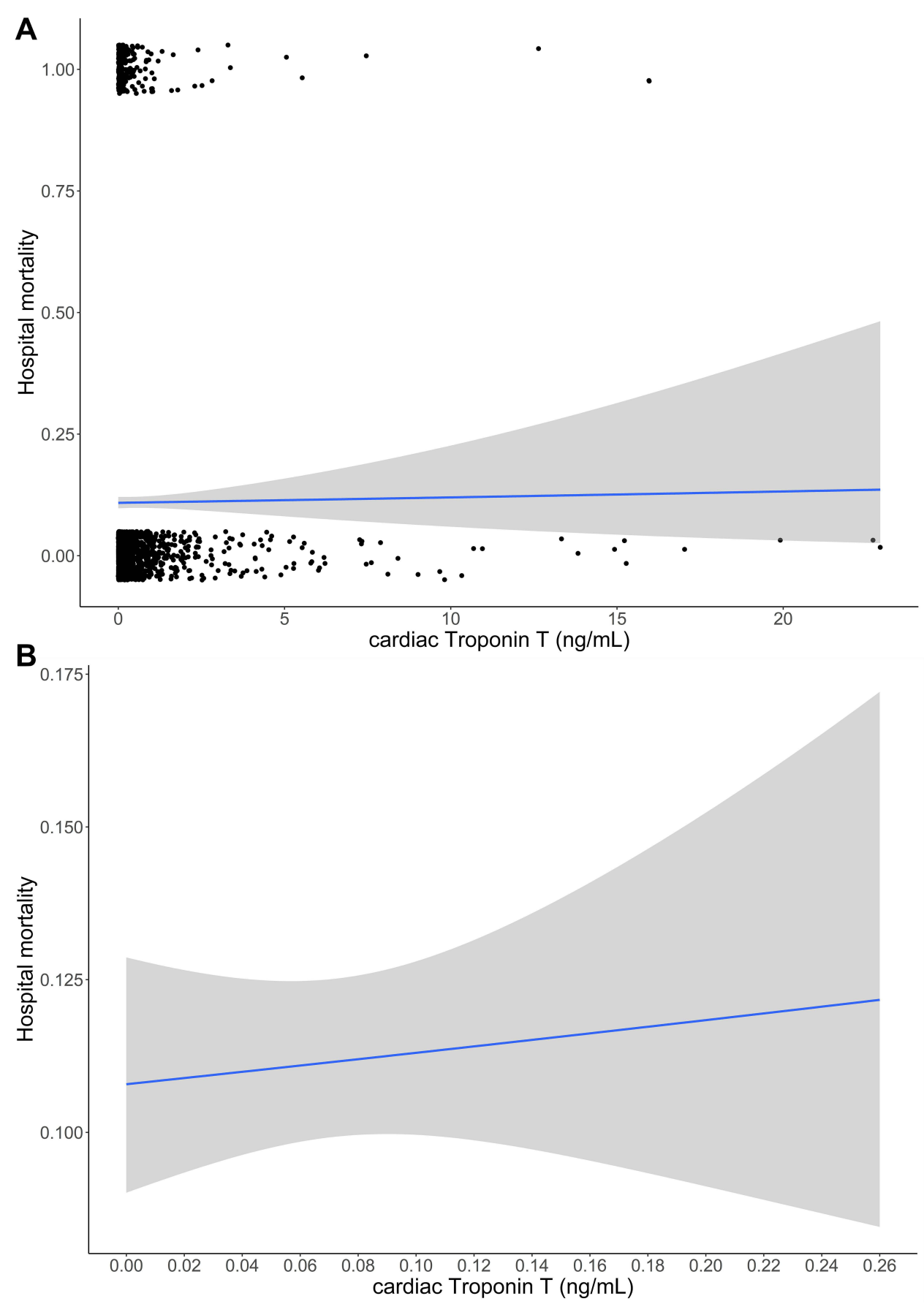

Figure 2 Relationship between cardiac Troponin T concentration on ICU admission and probability of hospital mortality.

Notes: (A) presents the relationship between cardiac Troponin T concentration and hospital mortality based on a univariable logistic regression model. (B) is the same as the $(\mathbf{A})$, but only cardiac Troponin T concentration within the range of $0.00-0.26 \mathrm{ng} / \mathrm{mL}$ is presented.

Abbreviation: ICU, intensive care unit.

who had a higher mortality rate. Landesberg et $\mathrm{al}^{19}$ investigated 101 high-cardiac-risk ICU patients and found $\mathrm{cTnT}$ elevation was associated with both early and late mortality. Gunnewiek et $\mathrm{al}^{20}$ investigated 34 ICU patients who were mechanically ventilated or underwent thoracic or vascular surgery and reported a cTnT- positive rate of $32 \%$, but there was no difference in mortality rates between cTnT-positive and cTnTnegative patients. Compared to these studies, we found a much higher proportion of cTnT elevation (92.23\%), which is also higher than studies that investigated $\mathrm{cTnI}$ $(21 \%-59 \%) .{ }^{12}$ This could be due to the different study 
Table 3 Association Between Concentration of Cardiac Troponin T on ICU Admission and Hospital Mortality

\begin{tabular}{|c|c|c|c|c|c|c|c|c|c|}
\hline \multirow{2}{*}{$\begin{array}{l}\text { Concentration of } \\
\text { Cardiac Troponin T on } \\
\text { ICU Admission* }\end{array}$} & \multicolumn{3}{|l|}{ Crude } & \multicolumn{3}{|l|}{ Model I** } & \multicolumn{3}{|l|}{ Model 2*** } \\
\hline & Odds Ratio & $95 \% \mathrm{Cl}$ & $\mathbf{P}$ & Odds Ratio & $95 \% \mathrm{Cl}$ & $\mathbf{P}$ & Odds Ratio & $95 \% \mathrm{Cl}$ & $\mathbf{P}$ \\
\hline $\begin{array}{l}\text { Two categories }(\mathbf{n g} / \mathbf{m L}) \\
\leq 0.01 \text { (normal) } \\
>0.01 \text { (abnormal) }\end{array}$ & $\begin{array}{l}\text { I (Reference) } \\
\text { I.58 }\end{array}$ & $0.95-2.63$ & 0.077 & $\begin{array}{l}\text { I (Reference) } \\
\text { I.39 }\end{array}$ & $0.8 \mathrm{I}-2.37$ & 0.228 & $\begin{array}{l}\text { I (Reference) } \\
\text { I.50 }\end{array}$ & $0.88-2.57$ & 0.140 \\
\hline $\begin{array}{l}\text { Five categories }(\mathbf{n g} / \mathbf{m L}) \\
\quad \leq 0.01 \\
0.02-0.04 \\
0.05-0.08 \\
0.09-0.26 \\
\geq 0.27\end{array}$ & $\begin{array}{l}\text { I (Reference) } \\
1.52 \\
1.89 \\
1.61 \\
1.40\end{array}$ & $\begin{array}{l}0.88-2.60 \\
1.09-3.30 \\
0.93-2.78 \\
0.8 I-2.44\end{array}$ & $\begin{array}{l}0.131 \\
0.024 \\
0.089 \\
0.230\end{array}$ & $\begin{array}{l}\text { I (Reference) } \\
1.39 \\
1.67 \\
1.27 \\
1.31\end{array}$ & $\begin{array}{l}0.78-2.44 \\
0.93-3.00 \\
0.7 I-2.25 \\
0.73-2.36\end{array}$ & $\begin{array}{l}0.261 \\
0.086 \\
0.423 \\
0.360\end{array}$ & $\begin{array}{l}\text { I (Reference) } \\
\text { I. } 44 \\
1.71 \\
1.36 \\
1.58\end{array}$ & $\begin{array}{l}0.8 I-2.56 \\
0.95-3.09 \\
0.76-2.43 \\
0.87-2.87\end{array}$ & $\begin{array}{l}0.209 \\
0.074 \\
0.302 \\
0.133\end{array}$ \\
\hline
\end{tabular}

Notes: *The maximum cardiac Troponin T concentration of available measurement records of cardiac Troponin T examined within 24 hours before and after the date of first ICU admission; **Model I was adjusting for age, sex, ethnicity, Simplified Acute Physiology Score II on ICU admission, and Charlson Comorbidity Index; ***Model 2 was adjusting for Model I and types of ICU. The type of ICU was determined by the first ICU admission if a patient had more than I ICU admission during the hospitalization. Abbreviations: ICU, intensive care unit; $\mathrm{Cl}$, confidence interval.

populations and the very limited sample sizes in these available studies (ie, chance findings). This speculation is supported by the findings from a prospective study that investigated a similar study population to ours (ie, ICU patients admitted for non-cardiac reasons), in which $84 \%(121 / 144)$ patients had elevated cTnT concentration $(\geq 0.015 \mathrm{ng} / \mathrm{mL}) .^{21}$

For the association of elevated cTnT concentration with prognosis, most of the above-mentioned studies

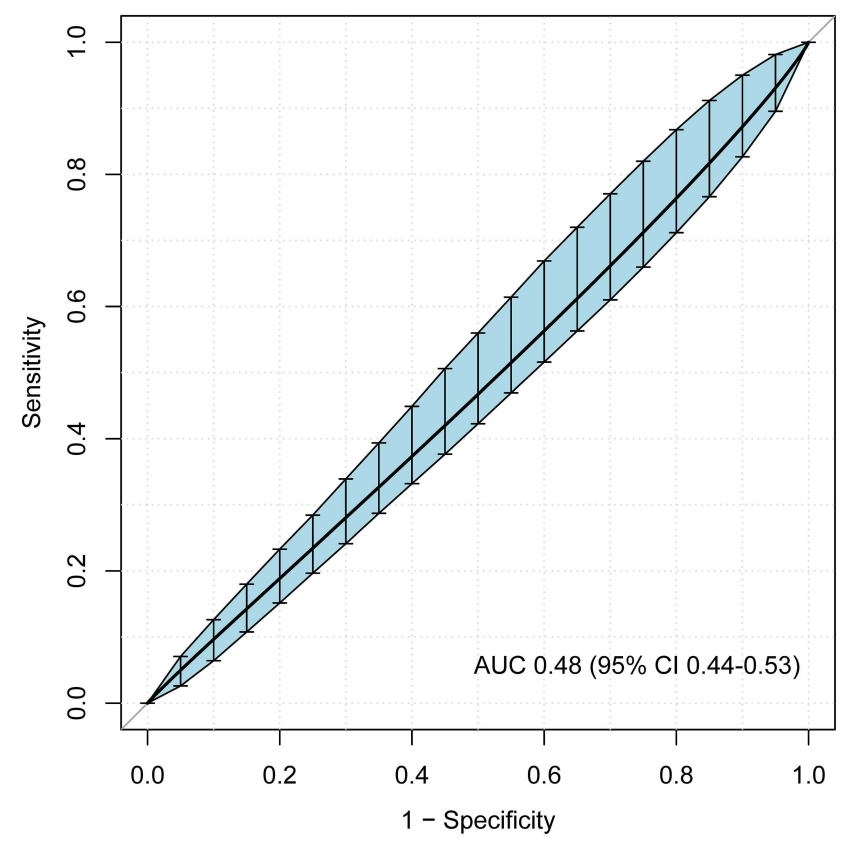

Figure 3 Receiver operating characteristic curve for cardiac Troponin T concentration ICU on admission to predict hospital mortality.

Abbreviations: ICU, intensive care unit; AUC, area under the receiver operating characteristic curve; $\mathrm{Cl}$, confidence interval. observed a higher mortality rate in patients with elevated cTnT concentration when compared to those with normal cTnT concentration. This is consistent with our findings, although the difference in hospital mortality rates was with a borderline $\mathrm{P}$ value $(\mathrm{P}=0.075)$. The above-mentioned studies are with limited sample sizes, but most of them detected a statistically significant difference in survival. A possible explanation is that these studies included patients with myocardial infarction, a population in which $\mathrm{cTnT}$ concentration has been proved to be with strong prognostic value. ${ }^{1,9}$ Unlike investigations on $\mathrm{cTnT}$, there are relatively more studies that investigated the prognostic value of $\mathrm{cTnI}$ in critically ill patients, ${ }^{12}$ but patients with myocardial infarction were also included in these studies. ${ }^{22,23}$

Except for the different study populations, it should also be noted that the majority of these available studies investigated the association simply by a direct comparison, which may be due to the limited sample sizes that made an adjusted analysis impossible. When the study population was stratified as five categories according to cTnT concentration, we observed that patients with a cTnT concentration of $0.05-0.08 \mathrm{ng} / \mathrm{mL}$ was statistically significantly associated with increased hospital mortality when compared to those with a normal cTnT concentration (crude OR 1.89, 95\% CI 1.09-3.30, $\mathrm{P}=0.024$ ). However, after adjusting for potential confounding including a disease severity score, the association was with a borderline $\mathrm{P}$ value (adjusted OR 1.71, 95\% CI 0.953.09, $\mathrm{P}=0.074)$. This suggests the observed increased risk of hospital mortality associated with elevated cTnT 
concentration may be attributed to the confounding. In our study, we observed a statistically significant higher prevalence of congestive heart failure, higher SAPS II and higher SOFA in patients with elevated cTnT concentration compared to those with normal cTnT concentration. This was consistent with the findings in the study conducted by Elst et $\mathrm{al},{ }^{24}$ that in a population with early septic shock, cTnI-positive patients presented a higher Acute Physiology and Chronic Health Evaluation II score and a higher prevalence of left ventricular dysfunction without evidence of acute ischemia. We also evaluate the predictive performance of cTnT concentration for hospital mortality, but a poor discrimination capacity (AUC 0.48, 95\% CI 0.44-0.53) was observed. In the study conducted by Crewdson et $\mathrm{al}^{25}$ which included 243 ICU patients with major trauma, the AUC for cTnT concentration to predict hospital mortality was 0.73 (95\% CI $0.65-0.80)$, but the study population did not exclude patients with direct myocardial injury.

Taken together, our findings suggest in critically ill patients without myocardial infarction, the coexisting comorbidities (such as heart failure, sepsis) or a severe condition may lead to an elevation of cTnT concentration, and therefore these patients appeared to have poor prognosis, but due to the poor discrimination capacity, our findings do not support to use cTnT concentration for prognostic risk stratification in this patient population. However, as suggested in guidelines, ${ }^{26}$ for patients with an elevated value of cTn in the absence of clinical evidence of ischemia, to searching for potential causes is suggested.

\section{Strengths and Limitations}

Our study has some strengths. First, for the first time the association between cTnT concentration on ICU admission and prognosis was investigated with a large sample size and adjusted analysis in the critically ill patients without myocardial infarction. Second, we included patients from different types of ICUs, which increases the generality of our findings. Third, our study was based on updated data (ie, including patients admitted to ICUs of a single center between 2008 and 2019).

Our study also has some limitations that should be noted when interpreting the results. First, the study is a retrospective study, and therefore all data we used for analysis were unvalidated. For example, information about how the cTnT concentration was measured was limited. All the diagnoses including myocardial infarction were identified by diagnosis codes, and we have no information about how myocardial infarction was screened in the study population. In addition, only patients with measurement records of cTnT concentration would be included in our study, which raised a concern about selection bias. Due to data limitation, indications for the examination of cTnT was unclear. In our study we observed that compared to normal cTnT concentration, patients with elevated cTnT concentration had a higher proportion of being admitted Coronary Care Unit (22.71\% versus 9.13\%). This suggests patients with suspected myocardial infarction might be more likely to receive the examination and therefore more likely to be included in our study. A prospective study is warranted to solve this problem. Second, we are unable to claim a causation given the nature of our study design. Ammann et $\mathrm{al}^{27}$ found in critically ill patients without acute coronary syndromes, elevated troponin is associated with decreased left ventricular function and higher levels of tumor necrosis factor-alpha and interleukin 6. Further investigations on the mechanisms behind the cTnT elevation in patients without acute coronary syndromes may provide more insights into this topic. Third, due to data limitation, only cTnT was investigated in our study, and future studies may investigate the prognostic value of cTnI in the study population. We only investigated the cTnT concentration on ICU admission, instead of its change. The study conducted by Hajsadeghi et $\mathrm{al}^{28}$ found an increase in cTnT concentration during ICU stay was associated with poor survival. Given the limited sample size, this should be confirmed in future studies. We only investigated short-term outcomes (ie, hospital mortality) in the study, while elevated cTnT concentration may be associated with poor long-term outcomes in ICU patients. $^{21,29}$

\section{Conclusions}

Elevated cTnT concentration on ICU admission appeared to be associated with poor prognosis in critically ill patients without myocardial infarction, but the association is not statistically significant after taking potential confounding into account. Together with a poor discrimination capacity, these results suggest that cTnT concentration has limited prognostic value in critically ill patients without myocardial infarction.

\section{Abbreviations}

cTn, Cardiac Troponin; ICU, intensive care unit; MIMIC IV, Medical Information Mart for Intensive Care IV; HIPAA, Health Insurance Portability and Accountability Act; CITI, Collaborative Institutional Training Initiative; ICD-9-CM, International Classification of Diseases, Ninth 
Revision, Clinical Modification; ICD-10-CM, International Classification of Diseases, Tenth Revision, Clinical Modification; LOINC, Logical Observation Identifiers Names and Codes; SAPS II, Simplified Acute Physiology Score II; GCS, Glasgow Coma Scale; SOFA, Sequential Organ Failure Assessment; CCI, Charlson Comorbidity Index; ROC, Receiver operating characteristic; AUC, area under the ROC curve; CI, confidence interval; OR, odds ratio.

\section{Data Sharing Statement}

The dataset analyzed during the current study is not publicly available due to the data use agreement, but request for access to the database is possible after following the necessary procedures (https://mimic-iv.mit.edu/docs/access/).

\section{Ethics Approval and Consent to Participate}

This study was exempt from institutional review board approval and patient consent due to the retrospective design, lack of direct patient intervention, and the security schema for the re-identification risk. The study complied with the Declaration of Helsinki.

\section{Funding}

This work was supported by the National Natural Science Foundation of China (grant No. 81670066), the Major Science and Technology Planning Project of Guangdong Province, China (grant No. 2016A020216009), and the Guangdong Basic and Applied Basic Research Foundation, China (grant No. 2019A1515011198). The funding sources were not involved in the study design, the collection, analysis and interpretation of data, the writing of the report, and the decision to submit the article for publication.

\section{Disclosure}

The authors report no conflicts of interest in this work.

\section{References}

1. Antman EM. Decision making with cardiac troponin tests. $N$ Engl $J$ Med. 2002;346(26):2079-2082. doi:10.1056/NEJMe020049

2. Braunwald E, Antman EM, Beasley JW, et al. ACC/AHA guidelines for the management of patients with unstable angina and non-ST-segment elevation myocardial infarction: executive summary and recommendations. A report of the American College of Cardiology/American Heart Association task force on practice guidelines (committee on the management of patients with unstable angina). Circulation. 2000;102 (10):1193-1209. doi:10.1161/01.cir.102.10.1193
3. Higgins JP, Higgins JA. Elevation of cardiac troponin I indicates more than myocardial ischemia. Clin Invest Med. 2003;26 (3):133-147.

4. $\mathrm{Wu} \mathrm{AH}$. Increased troponin in patients with sepsis and septic shock: myocardial necrosis or reversible myocardial depression? Intensive Care Med. 2001;27(6):959-961. doi:10.1007/s001340100970

5. Hijazi Z, Siegbahn A, Andersson U, et al. High-sensitivity troponin I for risk assessment in patients with atrial fibrillation: insights from the Apixaban for Reduction in Stroke and other Thromboembolic Events in Atrial Fibrillation (ARISTOTLE) trial. Circulation. 2014;129(6):625-634. doi:10.1161/CIRCULATIONAHA.113.006286

6. Horwich TB, Patel J, MacLellan WR, Fonarow GC. Cardiac troponin I is associated with impaired hemodynamics, progressive left ventricular dysfunction, and increased mortality rates in advanced heart failure. Circulation. 2003;108(7):833-838. doi:10.1161/01. CIR.0000084543.79097.34

7. Baillard C, Boussarsar M, Fosse JP, et al. Cardiac troponin I in patients with severe exacerbation of chronic obstructive pulmonary disease. Intensive Care Med. 2003;29(4):584-589. doi:10.1007/ s00134-003-1635-0

8. Dubin RF, Li Y, He J, et al. Predictors of high sensitivity cardiac troponin $\mathrm{T}$ in chronic kidney disease patients: a cross-sectional study in the chronic renal insufficiency cohort (CRIC). BMC Nephrol. 2013;14:229. doi:10.1186/1471-2369-14-229

9. Antman EM, Tanasijevic MJ, Thompson B, et al. Cardiac-specific troponin I levels to predict the risk of mortality in patients with acute coronary syndromes. $N$ Engl J Med. 1996;335(18):1342-1349. doi:10.1056/NEJM199610313351802

10. Willeit P, Welsh P, Evans JDW, et al. High-sensitivity cardiac troponin concentration and risk of first-ever cardiovascular outcomes in 154,052 participants. J Am Coll Cardiol. 2017;70(5):558-568. doi:10.1016/j.jacc.2017.05.062

11. Everett BM, Brooks MM, Vlachos HE, et al. Troponin and cardiac events in stable ischemic heart disease and diabetes. $N$ Engl $J$ Med. 2015;373(7):610-620. doi:10.1056/NEJMoa1415921

12. Lim W, Qushmaq I, Devereaux PJ, et al. Elevated cardiac troponin measurements in critically ill patients. Arch Intern Med. 2006;166 (22):2446-2454. doi:10.1001/archinte.166.22.2446

13. Relos RP, Hasinoff IK, Beilman GJ. Moderately elevated serum troponin concentrations are associated with increased morbidity and mortality rates in surgical intensive care unit patients. Crit Care Med. 2003;31(11):2598-2603. doi:10.1097/01.CCM.0000089931.09635. D2

14. Edouard AR, Felten ML, Hebert JL, Cosson C, Martin L, Benhamou D. Incidence and significance of cardiac troponin I release in severe trauma patients. Anesthesiology. 2004;101 (6):1262-1268. doi:10.1097/00000542-200412000-00004

15. Martin M, Mullenix P, Rhee P, Belzberg H, Demetriades D, Salim A. Troponin increases in the critically injured patient: mechanical trauma or physiologic stress? J Trauma. 2005;59(5):1086-1091. doi:10.1097/01.ta.0000190249.19668.37

16. Johnson A, Bulgarelli L, Pollard T, Horng S, Celi LA, Mark R. MIMIC-IV (version 0.4). 2020. Available from: https://doi.org/10. 13026/a3wn-hq05. Accessed March 8, 2021.

17. Charlson M, Szatrowski TP, Peterson J, Gold J. Validation of a combined comorbidity index. J Clin Epidemiol. 1994;47 (11):1245-1251. doi:10.1016/0895-4356(94)90129-5

18. Spies C, Haude V, Fitzner R, et al. Serum cardiac troponin $T$ as a prognostic marker in early sepsis. Chest. 1998;113(4):1055-1063. doi:10.1378/chest.113.4.1055

19. Landesberg G, Vesselov Y, Einav S, Goodman S, Sprung CL, Weissman C. Myocardial ischemia, cardiac troponin, and long-term survival of high-cardiac risk critically ill intensive care unit patients. Crit Care Med. 2005;33(6):1281-1287. doi:10.1097/01. CCM.0000166607.22550.87 
20. Klein Gunnewiek JMT, van de Leur J. Elevated troponin $\mathrm{T}$ concentrations in critically ill patients. Intensive Care Med. 2003;29(12):2317-2322. doi:10.1007/s00134-003-1953-2

21. Ostermann M, Lo J, Toolan M, et al. A prospective study of the impact of serial troponin measurements on the diagnosis of myocardial infarction and hospital and six-month mortality in patients admitted to ICU with non-cardiac diagnoses. Crit Care. 2014;18(2): R62. doi:10.1186/cc13818

22. Docherty AB, Sim M, Oliveira J, et al. Early troponin I in critical illness and its association with hospital mortality: a cohort study. Crit Care. 2017;21(1):216. doi:10.1186/s13054-017-1800-4

23. Reynolds T, Cecconi M, Collinson P, Rhodes A, Grounds RM, Hamilton MA. Raised serum cardiac troponin I concentrations predict hospital mortality in intensive care unit patients. $\mathrm{Br} J$ Anaesth. 2012;109(2):219-224. doi:10.1093/bja/aes141

24. Ver Elst KM, Spapen HD, Nguyen DN, Garbar C, Huyghens LP, Gorus FK. Cardiac troponins I and T are biological markers of left ventricular dysfunction in septic shock. Clin Chem. 2000;46 (5):650-657. doi:10.1093/clinchem/46.5.650
25. Crewdson K, Thompson J, Thomas M. Cardiac troponin T is associated with mortality in patients admitted to critical care in a UK major trauma centre: a retrospective database analysis. J Intensive Care Soc. 2019;20(2):132-137. doi:10.1177/1751143718767782

26. Thygesen K, Alpert JS, Jaffe AS, et al. Third universal definition of myocardial infarction. J Am Coll Cardiol. 2012;60(16):1581-1598. doi:10.1016/j.jacc.2012.08.001

27. Ammann P, Maggiorini M, Bertel O, et al. Troponin as a risk factor for mortality in critically ill patients without acute coronary syndromes. J Am Coll Cardiol. 2003;41(11):2004-2009. doi:10.1016/S0735-1097(03)00421-2

28. Hajsadeghi S, Gholami S, Gohardehi G, et al. Association between troponin T and ICU mortality, a changing trend. Cardiovasc J Afr. 2012;23(4):186-190. doi:10.5830/CVJA-2011-034

29. deFilippi CR, Tocchi M, Parmar RJ, et al. Cardiac troponin T in chest pain unit patients without ischemic electrocardiographic changes: angiographic correlates and long-term clinical outcomes. J Am Coll Cardiol. 2000;35(7):1827-1834. doi:10.1016/S07351097(00)00628-8
International Journal of General Medicine

\section{Publish your work in this journal}

The International Journal of General Medicine is an international, peer-reviewed open-access journal that focuses on general and internal medicine, pathogenesis, epidemiology, diagnosis, monitoring and treatment protocols. The journal is characterized by the rapid reporting of reviews, original research and clinical studies
Dovepress

across all disease areas. The manuscript management system is completely online and includes a very quick and fair peer-review system, which is all easy to use. Visit http://www.dovepress.com/ testimonials.php to read real quotes from published authors. 